\title{
How do we change practice when we have the evidence?
}

Janice P Burke

Thomas Jefferson University

Laura N. Gitlin

Follow this and additional works at: https://jdc.jefferson.edu/otfp

Part of the Occupational Therapy Commons

Let us know how access to this document benefits you

\section{Recommended Citation}

Burke, Janice P and Gitlin, Laura N., "How do we change practice when we have the evidence?" (2012). Department of Occupational Therapy Faculty Papers. Paper 53.

https://jdc.jefferson.edu/otfp/53

This Article is brought to you for free and open access by the Jefferson Digital Commons. The Jefferson Digital Commons is a service of Thomas Jefferson University's Center for Teaching and Learning (CTL). The Commons is a showcase for Jefferson books and journals, peer-reviewed scholarly publications, unique historical collections from the University archives, and teaching tools. The Jefferson Digital Commons allows researchers and interested readers anywhere in the world to learn about and keep up to date with Jefferson scholarship. This article has been accepted for inclusion in Department of Occupational Therapy Faculty Papers by an authorized administrator of the Jefferson Digital Commons. For more information, please contact: JeffersonDigitalCommons@jefferson.edu. 


\title{
THE ISSUE IS...
}

\section{How Do We Change Practice When We Have the Evidence?}

\author{
Janice Posatery Burke, Laura N. Gitlin
}

KEY WORDS

- health plan implementation

- occupational therapy

- patient care management

- professional practice

- translational medical research

Janice Posatery Burke, PhD, OTR/L, FAOTA, is Professor and Chair, Department of Occupational Therapy, and Dean, Jefferson School of Health Professions, Thomas Jefferson University, 901 Walnut Street, 6th Floor Philadelphia, PA 19107; Janice. burke@jefferson.edu

Laura N. Gitlin, PhD, is Professor, Department of Health Systems and Outcomes, School of Nursing, and Director, Changing Aging: Center for Innovative Interventions, Johns Hopkins University, Baltimore.

Translating research findings into practice includes myriad pragmatic realities, including understanding the suitability of the data to a particular patient group, writing new guidelines for occupational therapy practitioners, facilitating adoption of the guidelines, and instituting new patterns of care for patients. The process is more than a matter of disseminating the information to practitioners and expecting immediate change in patient treatment. Indeed, the field of implementation science is devoted to the identification of the numerous barriers and supports that constrain or expedite practice change in response to research. Moving forward and adopting evidence-based findings will require a focused understanding of the particular setting where change is warranted. Among the issues to address are the health system levels involved in change (professional, legislative, administrative, practitioner, and patient and family members), the values and beliefs of the participants, and knowledge of the communication channels that exist in the setting and how information and new ideas make their way through the setting.

Burke, J. P., \& Gitlin, L. N. (2012). The Issue Is - How do we change practice when we have the evidence? American Journal of Occupational Therapy, 66, e85-e88. http://dx.doi.org/10.5014/ajot.2012.004432

We envision that occupational therapy is a powerful, widely recognized, science-driven, and evidence-based profession with a globally connected and diverse workforce meeting society's occupational needs. (American Occupational Therapy Association [AOTA], 2007, p. 613)

In occupational therapy, an active cadre of researchers and practitioners is engaged in systematic studies examining the effectiveness of assessment and intervention for targeted populations of patients. This issue of the American Journal of Occupational Therapy describes some of those efforts. But what has happened, and what will happen, as these studies are completed and the evidence is available for occupational therapy practitioners to use in their practices? Will we be able to translate the findings into useful intervention protocols? Will we be able to train practitioners to deliver these interventions? Will practitioners be ready and willing to shift practice patterns? Will we be able to convince policymakers, administrators, and third-party payers that the interventions are worthwhile? Will patients and their families ac- cept new approaches that may conflict or compete with their expectations? In this column, we examine some of the key factors that we believe must be considered as occupational therapy practitioners face issues of adopting new evidence and other innovations to their practices.

Among the challenges facing occupational therapy and other health care professions steeped in the business of generating and using evidence are questions about how new, science-based breakthroughs find their way to practice. The process of developing, testing, and implementing research-based interventions is complex, costly, and time consuming. Establishing efficacy is one piece of the puzzle: "Efficacy trials are characterized by strong control in that a standardized program is delivered in a uniform fashion to a specific, often narrowly defined, homogeneous target audience" (Glasgow, Lichtenstein, \& Marcus, 2003, p. 1261). Effectiveness or intervention trials "to determine whether an intervention works among a broadly defined population" are another important feature (Glasgow et al., 2003, p. 1261). Effectiveness follows a predictable set of stages as outlined, for example, by Greenwald and 
Cullen (1985) in their call for a "national strategy to translate the rapid progress in basic knowledge about cancer into nationwide benefits" (p. 543) and by Gitlin, Vause-Earland, Piersol, and Shaw (2010), a group of researchers and occupational therapy practitioners, in their discussion of their own experience in translating occupational therapy research findings into a standard program of home-based care for older adults. The stages of establishing effectiveness include initial problem identification and hypothesis development, methods development, controlled intervention efficacy trials, effectiveness studies, translation to broad dissemination, and sustainability.

\section{Need for New Communication Channels}

Researchers share the common goal of identifying problems and conducting research studies that will produce findings that apply to populations in need and reach them in a timely way. But intense, high-stake challenges face researchers seeking ways to ensure that research findings make their way to the practice arena. Navigating the byways from "bench to bedside" (or, in our case, clinics, communities, homes, classrooms, and academic curricula) requires a multitiered "all hands on deck" approach that may not be commonplace among typically unconnected channels of professional communication (researchers, administrators, practitioners).

If we are going to take on the complicated task of seeding innovation in practice, then researchers, administrators, practitioners, and others must work collaboratively. We must participate in open exchanges as we develop, test, and refine all aspects of a randomized trial as it makes its way to clinical implementation. Participants must ask questions pertinent to practice such as the following: Is this a patient-based problem that would benefit from new information and change? Would practitioners, administrators, and patients value the change? Is there a perceived need for this particular type of innovation? Will practitioners have enough time to complete the assessments that are required and the treatment that is recommended?
Of equal weightiness are the practitioneroriented issues associated with moving evidence into practice. How do we cope with the new demands to be evidence based? How do we stay informed and also influence other members of the team and the administration to facilitate adoption of new ways of addressing old problems? How do we prepare ourselves as practitioners and the places where we work for the surge of new information and changes in techniques, patterns, and actions that follow the production of new effectiveness data? How do we involve administrators in the discussion about the value of establishing new services for patients and families in relation to the bottom line?

Today's health care environments and communities are rapidly changing and significantly challenging, with productivity pressures, increasing emphasis on the use of evidence to inform clinical decisions, and data-defying patient problems being the realities of daily practice. Where evidence fits may very well be dependent on the climate and culture of the practice site, which includes the values and beliefs of the occupational therapy practitioners and other workers in the immediate environment, of the referral sources, and of the patients, clients, and families who are the target of the intervention being undertaken.

It is clear that practitioners need and want to know how they can deliver the best care possible. How exactly they receive, process, and use that information remains another question. To understand how research makes its way to readiness for consumers and practitioners and how new information becomes a part of the practitioner's perspective and practice, we need to take a careful look at the influences and patterns of change that affect one another.

\section{Establishing the Players}

In an analysis of implementation in nursing research, Helfrich and colleagues (2010) used the PARIHS (Promoting Action on Research in Health Services) framework to assess how evidence moves into practice. These authors identified a broad cadre of organizational, professional, and individual constituents and factors in the evidence-based arena that play a part in the successful implementation of evidence in practice. In similar efforts, Kitson and colleagues (2008) addressed the obstacles to adoption of innovations and found "a lack of a true appreciation or understanding of the multiple factors involved" in the "spread of best practice" (p. 2). They called for

a shift away from the traditional notion that getting evidence into practice is straightforward. Until relatively recently the spread of evidence was seen as a linear and technical process at the level of the individual, and was described as changes in clinicians' behavior in line with evidence-based guidelines. Now there is widespread recognition that ... implementation ... requires whole system change. (p. 2)

When we think about health care organizations as multitiered systems, we can envision the types of components that need to be assessed at each level to instigate change. At the very grandest level are policy imperatives established in Congress and health care organizations articulating the mandate of evidence-based practice (EBP).

Trickling down to the next level, regulators establish guidelines for health care agencies and third-party payers. At this point, hospitals and clinics establish an array of mechanisms, including clinical pathways, intervention guidelines, and protocols, to designate the criteria or threshold for treatment and the frequency and dosage for diagnostic categories in a drive toward using evidence to support or refute the benefit of treatment. Other participants, such as professional organizations across the spectrum of health care, establish mechanisms for practitioners, consumers, referral sources, educators, and students to have ready access to materials and resources addressing the availability of evidence (e.g., AOTA evidence-based briefs). Similarly, accreditation bodies (in our case, the Accreditation Council on OccupationalTherapy Education) establish educational standards that are incorporated into all accredited professional programs delivering entry education to ensure that students learn and practice essential entrylevel skills. 
When research is involved, it is imperative for researchers and academics to partner with practicing occupational therapists, agency administrators, and patients and families to develop strategies that will increase the use or transfer of evidence into practice. Similarly, these groups will need to become versed in the issues and strategies associated with implementation to support research that generates evidence that is practice relevant.

Several authors have facilitated, interviewed, or surveyed people involved in knowledge transfer (Conklin \& Stolee, 2008; Gabbay \& le May, 2011), including occupational therapists, to understand more about the experience of using evidence in practice (Coster \& Schwarz, 2004; Dysart \& Tomlin, 2002; Dubouloz, Egan, Vallerand, \& von Zweck, 1999).

These responses can inform questions to be asked and concerns to be addressed when moving toward implementation. Additionally, Prochaska and Velicer's (1997) work on the five stages of behavioral change (precontemplation, contemplation, preparation, action, and maintenance) provides important insights about readiness to consider change as we move to new ways of knowing and practicing evidence-based occupational therapy within the greater system of health and community care.

\section{Knowing More About the Complexity of Implementation}

We have established that EBP is not a simple matter of informing practitioners that new information exists that they should use to inform their practices. Implementation of EBP "requires multiple strategies to address the nature of the EBP topic, the manner in which the evidence is communicated to those who deliver care, and the context in which they work" (Titler, Everett, \& Adams, 2007, p. S53). Indeed, many have devoted attention to implementation science, which has been defined as

the investigation of methods, interventions (strategies), and variables to influence adoption of evidence-based healthcare practices by individuals and organizations to improve clinical and operational decision making [including] testing the effectiveness of interventions to promote and sustain use of evidence-based healthcare practices. (Titler et al., 2007, p. S53)

We can learn from projects under way in nursing that are looking at how nurses and their teams increase their research use with inclusion of "organizational factors in addition to individual factors that support and thwart it" (Titler et al., 2007, p. S54). The nursing literature supports the ideas in this column, namely that translating researchinto practice intervention is practitioner sensitive and site and patient population specific. These ideas are critical for occupational therapy as we move toward increased research use in our field.

\section{Mindlines: How Clinicians Adopt New Ideas}

How clinical knowledge passes into the everyday world of clinical practice at the hands of occupational therapists is of equal consideration. In a study of key factors in the adoption of new information, Helfrich, Li, Sharp, and Sales (2009) named three core elements that influence a person's way of thinking: evidence, knowledge, and context. Successful acceptance of new information depends on the presence of and interaction among the three elements of influence. These influences seem equally appropriate for consideration in an occupational therapy scenario. A fascinating ethnographic study by Gabbay and le May (2011) illustrates this point.

Gabbay and le May (2011) followed general practitioners in the United Kingdom as they went about their work conducting office, home, and nursing clinic visits; documenting patient charts; attending practice meetings; and monitoring and watching over the administration of their practice. The researchers observed, interviewed, reviewed documents, completed member checks, and so forth. In an effort to understand how evidence makes its way into practice, these ethnographers focused their attention on how formal knowledge shapes practitioners' ways of thinking and the strategies they use to absorb new knowledge, call on it, and use it. Their work will be very familiar to occu- pational therapists, who are versed in clinical reasoning. As one would expect, the practitioners used their clinical knowledge to make patient decisions, but Gabbay and le May found that they also "blend formal, informal, tactic and experiential evidence into signposts for action" (p. xiii). They coined the term mindlines to describe this complex and reflective process. Mindlines evolve on the basis of information acquisition, application, and use and varying contexts of practice (Gabbay \& le May, 2011, p. xiii). But what about new knowledge that must be learned from new research evidence? Gabbay and le May found that this new knowledge has a "social life" of its own and makes its way into clinicians' mindlines in unique, contextually based ways.

All occupational therapy clinical practitioners learn a great deal of information from a wide variety of sources, including informal interactions with other professionals. They rely on one another to share information from sources such as continuing education programs, lectures and learning opportunities with experts, material scanned and read in the professional literature, insights gained from telling patient stories and sharing clinical insights, and the Internet. In each work setting, the social life of knowledge follows a distinct line of information flow as participants identify what may be useful research evidence and consider it in relation to their clinical experience and the patient populations being served. Given the complexity of this task, it is clear that some settings and contexts will be more conducive to successful implementation than others.

\section{Starting the Conversation}

So where do we begin? The process of implementation requires a systemwide approach that must be customized to a given setting and context. We offer the following ideas to serve as a starting point for initiating a process that will evolve to fit the needs of each individual clinical site.

At colleges and universities, faculty and students are deeply embroiled in the task of understanding the methods for collecting and analyzing evidence for a wide range of assessment and treatment scenarios. They have the skills and resources to troll databases, 
access the most current information, and put it into understandable formats.

It is common for an academic-based occupational therapy program to require students to complete evidence-based assignments as part of course requirements. Many students are involved in these types of assignments while they are on their fieldwork rotations or after they have just completed them, using the patient problems they have come to know to develop questions about evidence. Developing partnerships between clinical programs and academic programs is a first step in clinician access to the evidence that student and faculty have amassed. Some suggestions for forming such partnerships include the following:

- Invite faculty from your local occupational therapy academic program to your clinic and initiate discussions about the patient assessment and treatment problems that are of high interest in your clinic.

- Talk about ways you can contribute to the efforts of students as they select evidence-based topics that would be of mutual interest.

- Engage clinicians, administrators, staff, and patients in discussions about what they would like to know more about and focus on as institution "brands."

Using one of these suggestions as a first step will facilitate your ability to work with others to move relevant evidence into everyday practice on the basis of the specific dynamics, resources, and opportunities at your site.

At the clinical level, other suggestions include the following:

- Complete thorough analyses of the culture and values of the settings, recognize the important stakeholders and decision makers, and approach them to discuss your staff s interest in implementing evidence in the offerings of the clinic.

- Understand your position in the system, enlist support, deconstruct obstacles, and carefully manage concerns and resources that are influencing your outcomes. These steps will contribute to the direction of the project you are about to undertake.
Understanding how decisions are made, how priorities are set, and where the drivers of change are situated while understanding the social life of knowledge and its value in your particular setting will significantly increase your ability to adopt new ideas and seed innovations by all of the members of the community.

\section{Moving Forward}

As we move forward in our discovery of the science of and evidence for occupational therapy, it is imperative that we fully prepare for success and continued growth across all constituent groups: students and educators, new and seasoned clinicians, researchers, agency and school administrators, policy and regulatory personnel, patients and families, and referral sources. With a heightened awareness of the move toward EBP, practitioners must contribute to a healthy discourse outlining the challenges of implementation at and across all levels (policy, profession, health delivery organization, individual practitioner). The health promotion, health care reform, and health professions literatures and the implementation science that has already appeared in journals and newsletters provide an appropriate start point. In the effort to increase the adoption of evidencebased programs and intervention methods, our professional organizations, academic programs, educators, and clinicians all need to be involved. We look forward to the exciting opportunities and challenges that translation of research into practice presents as we move toward the goals of AOTA's (2007) Centennial Vision.

\section{References}

American Occupational Therapy Association. (2007). AOTA's Centennial Vision and executive summary. American Journal of Occupational Therapy, 61, 613-614. http://dx.doi. org/10.5014/ajot.61.6.613

Conklin, J., \& Stolee, P. (2008). A model for evaluating knowledge exchange in a network context. Canadian Journal of Nursing Research, 40, 116-124.

Coster, W., \& Schwarz, L. (2004, June). Facilitating transfer of evidence-based practice into practice. Education Special Interest Section Quarterly, 14, 1-3.
Dubouloz, C.-J., Egan, M., Vallerand, J., \& von Zweck, C. (1999). Occupational therapists' perceptions of evidence-based practice. American Journal of Occupational Therapy, 53, 445-453.

Dysart, A. M., \& Tomlin, G. S. (2002). Factors related to evidence-based practice among U.S. occupational therapy clinicians. American Journal of Occupational Therapy, 56, 275-284. http://dx.doi.org/10.5014/ajot.56.3.275

Gabbay, J., \& le May, A. (2011). Practice-based evidence for healthcare: Clinical mindlines. Abingdon, England: Routledge.

Gitlin, L., Vause-Earland, T., Piersol, C., \& Shaw, G. (2010). Enhancing quality of life in functionally vulnerable older adults: From randomized trial to standard care. Generations, 34, 84-87.

Glasgow, R. E., Lichtenstein, E., \& Marcus, A. C. (2003). Why don't we see more translation of health promotion research to practice? Rethinking the efficacy-toeffectiveness transition. American Journal of Public Health, 93, 1261-1267. http:// dx.doi.org/10.2105/AJPH.93.8.1261

Greenwald, P., \& Cullen, J. W. (1985). The new emphasis in cancer control. Journal of the National Cancer Institute, 74, 543-551.

Helfrich, C. D., Damschroder, L. J., Hagedorn, H. J., Daggett, G. S., Sahay, A., Ritchie, M., et al. (2010). A critical synthesis of literature on the Promoting Action on Research Implementation in Health Services (PARIHS) framework. Implementation Science; 5(82), 1-20. http://dx.doi.org/10.1186/1748-5908-5-82

Helfrich, C. D., Li, Y.-F., Sharp, N. D., \& Sales, A. E. (2009). Organizational Readiness to Change Assessment (ORCA): Development of an instrument based on the Promoting Action on Research in Health Services (PARIHS) framework. Implementation Science, 4(38), 1-13.

Kitson, A. L., Rycroft-Malone, J., Harvey, G., McCormack, B., Seers, K., \& Titchen, A. (2008). Evaluating the successful implementation of evidence into practice using the PARiHS framework: Theoretical and practical challenges. Implementation Science, 3(1), 1-12.

Prochaska, J. O., \& Velicer, W. F. (1997). The transtheoretical model of health behavior change. American Journal of Health Promotion, 12, 38-48. http://dx.doi.org/10.4278/ 0890-1171-12.1.38

Titler, M. G., Everett, L. Q., \& Adams, S. (2007). Implications for implementation science. Nursing Research, 56(Suppl.), S53-S59. http://dx.doi.org/10.1097/01. NNR.0000280636.78901.7f 